\title{
Operacionalização do modelo de avaliação de aprendizagem organizacional proposto por Igarashi (2009) em um polo de ensino a distância
}

\author{
Wagner Igarashi (UNIOESTE) wigarash@gmail.com \\ Deisy Cristina Corrêa Igarashi (UNIOESTE) deisyigarashi@gmail.com \\ Marina Keiko Nakayama (UFSC) marina@egc.ufsc.br
}

\begin{abstract}
Resumo
O estudo tem como objetivo operacionalizar o modelo de avaliação da aprendizagem organizacional proposto por Igarashi (2009) em um polo de ensino a distância da Universidade Aberta do Brasil. O estudo de caráter descritivo e qualitativo segue as etapas propostas por Igarashi (2009) e apresenta a estruturação e operacionalização do modelo, o qual atende aos anseios dos autores pesquisados por possibilitar, a partir da interação com os envolvidos, a construção de um instrumento de avaliação da aprendizagem que contemple elementos intrínsecos à organização. Como resultado a operacionalização do modelo gerou conhecimento ao gestor, em termos do desempenho local e global do polo, bem como a possibilidade de se analisar ações de melhorias que apóiem o gerenciamento da organização.
\end{abstract}

Palavras chave: Modelo, Avaliação, Aprendizagem organizacional.

\section{Application of organizational learning evaluation model proposed by Igarashi (2009) in a distance education pole}

\begin{abstract}
The study has as objective to apply the organizational learning evaluation model proposed by Igarashi (2009) in a distance education pole of the Universidade Aberta do Brasil. The study of descriptive and qualitative character follows the stages proposals for Igarashi (2009) and it presents the structuring and operating of the model, which contemplate the concerns of the authors researched by facilitating, starting from the interaction with involved them, the construction of an organizational learning evaluation instrument that contemplates the organization intrinsic elements. As result the model applying generated knowledge for the manager, in terms of local and global acting, as well as the possibility to analyze possible improvement actions that support the management of the organization.
\end{abstract}

Keywords: Model, Evaluation, Organizational Learning.

\section{Introdução}

As pesquisas desenvolvidas na área de aprendizagem organizacional buscam gerar subsídios, a fim de auxiliar na identificação de elementos que sejam capazes de apoiar as organizações, bem como no processo de se identificar elementos que devam ser observados no processo de avaliação. Neste sentido, Igarashi, Igarashi e Nakayama (2009) realizaram uma pesquisa teórica a fim de identificar quais elementos estão sendo considerados pelos autores no âmbito nacional e internacional, Quadro 1.

Quadro 1: Percepções informadas pelo contexto nacional e internacional

\begin{tabular}{|c|c|}
\hline Contexto nacional e internacional & Percepção identificada \\
\hline Dove (1999); Bollinger; Smith (2001); Huosong; & a aprendizagem organizacional deveria ser \\
Kuanqi; Shuqin (2003); Othman; Hashim (2004); & apoiada por ferramentas de tecnologia da \\
Falconer (2006); Mcadam; Mason; Mccrory (2007) & informação \\
\hline Dove (1999) & a aprendizagem organizacional se refere a um \\
\hline
\end{tabular}




\begin{tabular}{|c|c|}
\hline & processo de mudança cultural \\
\hline $\begin{array}{l}\text { Bollinger; Smith (2001); Mathiassen; Pourkomeylian } \\
\text { (2003); Loebbecke; Wareham (2003); Halawi; } \\
\text { Mccarthy; Aronson (2006); Meroño-Cerdan; Lopez- } \\
\text { Nicolas; Sabater-Sánchez (2007) }\end{array}$ & $\begin{array}{c}\text { a aprendizagem organizacional deveria } \\
\text { observar as especificidades organizacionais }\end{array}$ \\
\hline $\begin{array}{c}\text { Huosong; Kuanqi; Shuqin (2003); Loebbecke; } \\
\text { Wareham (2003); Nevo; Furneaux; Wand Othman; } \\
\text { Hashim (2004); Gasson; Shelfer (2007); Meroño- } \\
\text { Cerdan; Lopez-Nicolas; Sabater-Sánchez (2007); } \\
\text { Mcadam; Mason; Mccrory (2007) }\end{array}$ & $\begin{array}{c}\text { a possibilidade da tecnologia da informação } \\
\text { auxiliar no processo de aprendizagem } \\
\text { organizacional como um todo (alavanca, } \\
\text { conduz e direciona) }\end{array}$ \\
\hline Contexto internacional & Percepção identificada \\
\hline Bollinger e Smith (2001); Dove (1999) & $\begin{array}{l}\text { a cultura adequada encoraja o } \\
\text { compartilhamento do conhecimento }\end{array}$ \\
\hline Falconer (2006) & $\begin{array}{c}\text { as tecnologias e técnicas de } \text {-learning podem } \\
\text { encorajar e facilitar a aprendizagem } \\
\text { organizacional }\end{array}$ \\
\hline $\begin{array}{c}\text { Meso e Smith (2000); Szymczak e Walker (2003); } \\
\text { Chae e Lanzara (2006) }\end{array}$ & $\begin{array}{c}\text { as organizações precisam considerar não } \\
\text { apenas a tecnologia, mas também a infra- } \\
\text { estrutura organizacional, a cultura } \\
\text { organizacional e as pessoas }\end{array}$ \\
\hline Halawi, McCarthy e Aronson (2006) & $\begin{array}{c}\text { é preciso considerar cultura de } \\
\text { compartilhamento do conhecimento }\end{array}$ \\
\hline $\begin{array}{l}\text { Meroño-Cerdan, Lopez-Nicolas e Sabater-Sánchez } \\
\text { (2007) }\end{array}$ & $\begin{array}{c}\text { a estratégia de personalização se torna um } \\
\text { diferencial ao se formalizar a gestão do } \\
\text { conhecimento, principalmente nas primeiras } \\
\text { etapas onde são definidos os instrumentos que } \\
\text { apoiarão as estratégias e conduzirão a } \\
\text { mensuração }\end{array}$ \\
\hline $\begin{array}{c}\text { Meroño-Cerdan, Lopez-Nicolas e Sabater-Sánchez } \\
\text { (2007), Nevo, Furneaux e Wand (2007), Gasson e } \\
\text { Shelfer (2007) }\end{array}$ & $\begin{array}{c}\text { delinear a importância de instrumentos e } \\
\text { modelos de avaliação de sistemas de gestão do } \\
\text { conhecimento }\end{array}$ \\
\hline $\begin{array}{c}\text { Meroño-Cerdan, Lopez-Nicolas e Sabater-Sánchez } \\
\text { (2007), Nevo, Furneaux e Wand (2007), Gasson e } \\
\text { Shelfer (2007) }\end{array}$ & $\begin{array}{l}\text { a gestão do conhecimento deveria ser apoiada } \\
\text { por ferramentas de tecnologia da informação }\end{array}$ \\
\hline $\begin{array}{l}\text { Bollinger e Smith (2001), Halawi, McCarthy e } \\
\text { Aronson (2006), Mathiassen e Pourkomeylian (2003), } \\
\text { Loebbecke e Wareham (2003), Meroño-Cerdan, } \\
\text { Lopez-Nicolas e Sabater-Sánchez (2007) }\end{array}$ & $\begin{array}{l}\text { processo de aprendizagem organizacional/ } \\
\text { gestão do conhecimento deve estar alinhado à } \\
\text { estratégia de cada organização }\end{array}$ \\
\hline
\end{tabular}

Fonte: Adaptado de Igarashi, Igarashi e Nakayama (2009)

A partir dos elementos elencados no Quadro 1, Igarashi, Igarashi e Nakayama (2009) consideram viável a construção, de um modelo que avalie a aprendizagem organizacional. Os autores ainda observam que

[...] tal proposta é viável desde que sejam observadas as preocupações de Silva e Rozenfeld (2003), no contexto nacional e de Meso e Smith (2000), Bollinger e Smith (2001), Halawi, McCarthy e Aronson (2006), Chae e Lanzara (2006), Mathiassen e Pourkomeylian (2003), no contexto internacional; os quais fazem menção às características intrínsecas à organização de como esta pode encontrar seu próprio equilíbrio e amadurecimento (Igarashi, Igarashi E Nakayama, 2009).

Como limitação da pesquisa tem-se que o estudo foi desenvolvido utilizando a percepção no contexto internacional, restrito aos artigos consultados a partir do Portal de Periódicos da CAPES e no contexto nacional restrito ao portal de periódicos e artigos SCIELO. Por isso, podem haver percepções diferentes de outros autores que não foram considerados por Igarashi (2009) ao realizar a proposta teórica do modelo. Uma vez considerada esta limitação, o estudo tem por objetivo operacionalizar o modelo de 
avaliação da aprendizagem organizacional proposto por Igarashi (2009) em um polo de ensino a distância da Universidade Aberta do Brasil (UAB), do estado do Paraná. A partir da operacionalização do modelo busca-se responder ao seguinte questionamento: O modelo proposto por Igarashi (2009) demonstrou alinhamento com as especificidades organizacionais e apresentou elementos que auxiliem o polo a delinear sua estratégia?

Em relação ao objetivo o presente estudo utilizou a abordagem descritiva, ao se realizar a análise dos elementos que apóiam a estruturação e operacionalização do modelo proposto por Igarashi (2009). Além disso, o estudo utiliza a abordagem qualitativa, ao se proceder a análise da visão estratégica dos stakeholders do polo, objeto de estudo, por meio de entrevistas interativas em que se buscou o consenso dos envolvidos. As entrevistas subsidiaram a estruturação e personalização do modelo de avaliação da aprendizagem organizacional.

Além desta seção de caráter introdutório, este estudo é composto por mais quatro seções: seção 2 - apresenta os elementos que compõem o modelo de avaliação; seção 3 - apresenta o processo de estruturação e personalização do modelo; seção 4 - delineia-se a operacionalização do modelo, ou seja, sua estruturação e operacionalização; seção 5 são realizadas as considerações finais sobre a operacionalização e a análise de seus resultados. Por fim, são apresentadas as referências que embasam o estudo.

\section{Proposta do modelo}

A estruturação do modelo, nos moldes que se propõe neste estudo, pode auxiliar no processo de avaliação direcionado à aprendizagem organizacional. A estruturação e operacionalização do modelo fazem uso de aspectos fundamentais que direcionam um processo de avaliação, ou seja, "quem será avaliado", "qual será a finalidade da avaliação", "o que será avaliado", “como será avaliado” e "como conduzir o gerenciamento” (Miklós,1980; Igarashi et al., 2006). A estrutura do modelo considera a categorização dos indicadores a partir das percepções sobre aprendizagem organizacional e segundo o objetivo estratégico da organização. A Figura 1 ilustra o modelo proposto. As cinco percepções apresentadas foram extraídas a partir da hierarquização dos elementos apontados pelos autores pesquisados, como sendo pontos centrais no processo de avaliação da aprendizagem organizacional.

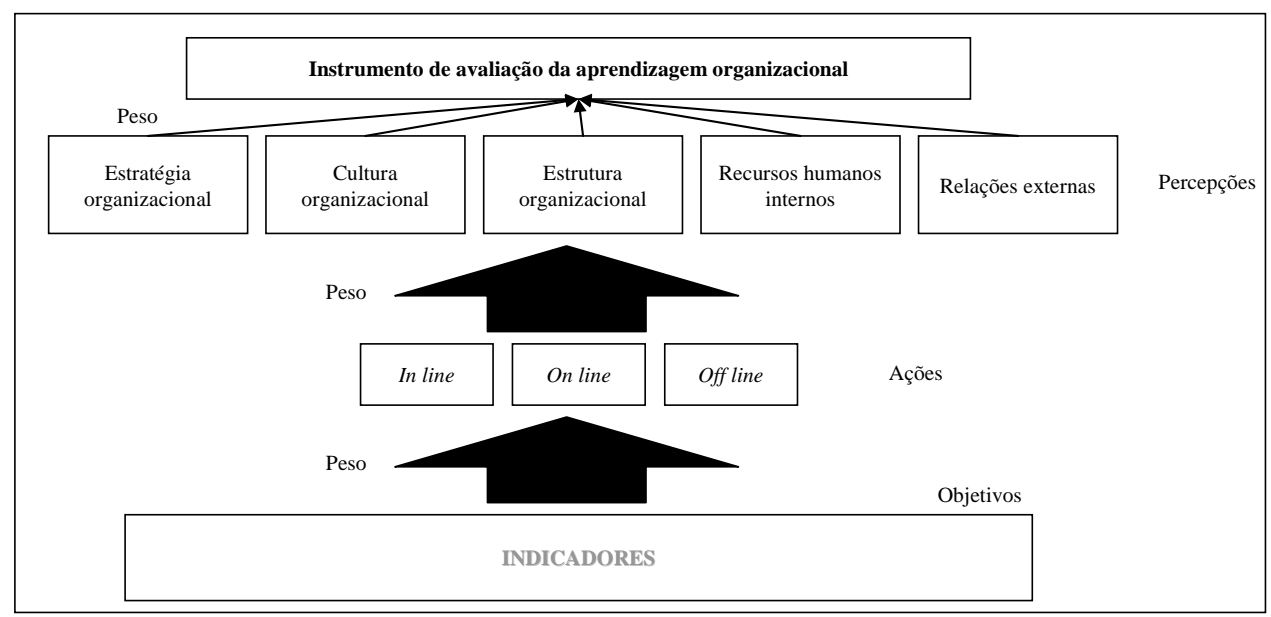

Figura 1: Ilustração do modelo de avaliação da aprendizagem organizacional proposto por Igarashi (2009)

Na Figura 1 o primeiro nível é referente ao desempenho global da organização. O nível seguinte refere-se às cinco percepções sobre aprendizagem organizacional (estratégia organizacional, cultura organizacional, estrutura organizacional, recursos 
humanos internos e relações externas), nas quais serão categorizadas as preocupações da organização. Nos níveis seguintes as preocupações são categorizadas em relação às ações estratégicas in-line, off-line ou on-line (operacional, tático ou estratégico) e em preocupações macro, caso haja necessidade, até se chegar aos objetivos. A partir dos objetivos são estruturados os indicadores, os quais se referem aos elementos que serão pontualmente objeto de avaliação. Após a estruturação e personalização do modelo, sua operacionalização tem início pelos indicadores, ou seja, em nível local, na parte inferior da Figura 1. Os indicadores são agrupados ao longo do modelo (de baixo para cima) a partir da atribuição de pesos até se atingir o nível global de desempenho.

Apresentado o modelo, segundo o enfoque teórico, passa-se a apresentar os elementos que informam o processo de estruturação e personalização do modelo.

\section{Elementos que informam o processo de estruturação e personalização do modelo}

Para o processo de estruturação do modelo foram definidas algumas fases. A apresentação das fases sintetiza os procedimentos a serem adotados, bem como explicita cada ação realizada ao estruturar e personalizar o modelo de avaliação direcionado à aprendizagem organizacional. Cada uma das etapas e fases, que compõem o processo de estruturação do modelo, é apresentada na Figura 2.

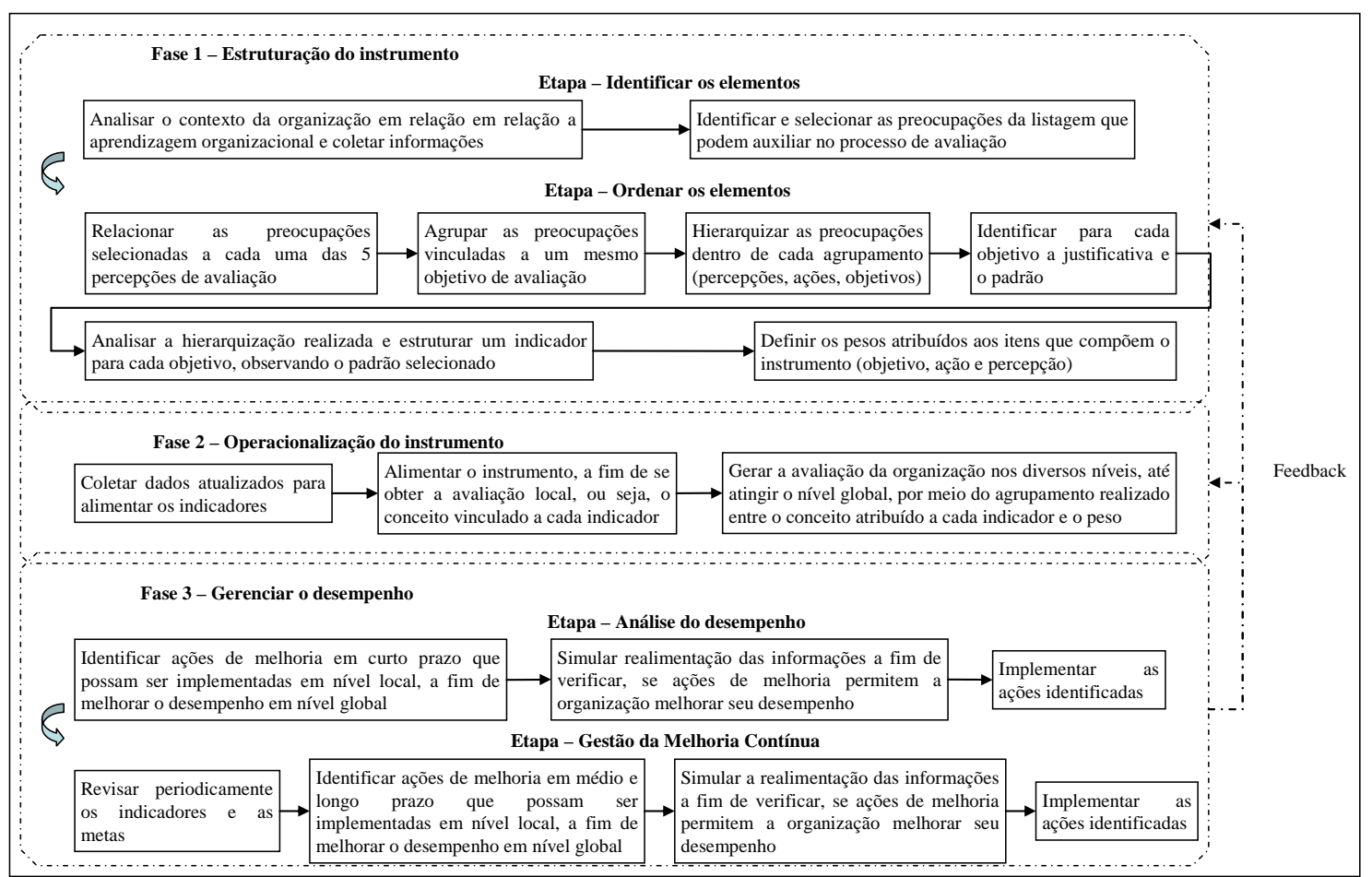

Figura 2: Processo de estruturação e personalização do modelo de avaliação proposto por Igarashi (2009)

Na Figura 2, a fase 1 coleta as informações que irão personalizar o modelo. Recomenda-se que a coleta e a análise das informações, sejam realizadas com rigor, para que os elementos essenciais sejam inseridos no processo. $\mathrm{Na}$ fase 2, são construídos os indicadores vinculados aos objetivos. Ainda na fase 2 se inicia a operacionalização do modelo. Salienta-se que o sucesso da fase 3 depende da fase 2, uma vez que o conceito local, aqui, atribuído é agrupado até o nível global do modelo. $\mathrm{O}$ agrupamento dos indicadores é realizado segundo as ações in-line, on-line ou off-line e sua correlação com as ações de planejamento. Com as informações resultantes do processo de avaliação local (indicadores) e global é possível iniciar a fase 3. Esta fase se 
refere ao processo de gestão. Ou seja, são identificadas possíveis ações de melhoria em curto prazo que possam ser implementadas em nível local, a fim de melhorar o desempenho em termos globais. Por fim, nesta fase é prevista a revisão periódica dos indicadores e das metas que compõem o modelo. Assim, o modelo proposto apresenta um ciclo permanente de melhoria contínua ao processo. Ainda em relação às fases 2 e 3 , o modelo gera a possibilidade de simular a realimentação das informações a fim de verificar, se ações de melhoria permitem às organizações melhorarem seus desempenhos da forma esperada, antes mesmo de tais ações sejam implementadas.

Salienta-se que o modelo proposto permite um processo de gestão flexível e contínuo. Uma vez que ele pode ser ajustado ou alterado conforme a necessidade da organização. Para tanto, basta que as etapas descritas sejam seguidas.

\section{Operacionalizado do modelo proposto}

A primeira aplicação do modelo foi realizada em um dos polos de ensino a distância da Universidade Aberta do Brasil (UAB), no estado do Paraná. O polo não autorizou a divulgação de sua denominação, por isso ele foi denominado no decorrer do estudo como POLO_EAD.

\subsection{Fase 1 - Estruturação do modelo}

Foi realizada uma pesquisa inicial sobre o tema UAB que possibilitou ao pesquisador conhecer aspectos quanto ao perfil e ao contexto do polo, objeto de estudo, para que se pudesse conhecer melhor a organização. Durante a fase de coleta de dados foi realizada uma reunião geral com os diversos membros da organização em estudo, o coordenador do polo e os servidores que realizam atividades de suporte e técnica, por serem os membros efetivos da organização. Como os participantes tinham pouca experiência sobre o assunto aprendizagem organizacional, lhes foi apresentada uma listagem de preocupações vinculadas à aprendizagem organizacional, obtida a partir das pesquisas teóricas sobre o tema, durante o processo de desenvolvimento do modelo.

A partir da listagem, os participantes puderam identificar elementos que pudessem compor o modelo (Figura 3 (a)). As preocupações selecionadas pelos diversos atores foram confrontadas e validadas pelo Coordenador do POLO_EAD. Em seguida elas foram categorizadas e hierarquizadas (Figura 3 (b)) em formato gráfico, observando a relação causa-efeito. Destaca-se que os elementos terminais da estrutura hierárquica (objetivos), são a base para a criação dos indicadores. E a partir destes foi realizada a identificação da justificativa e do padrão. Com a identificação da justificativa e do padrão vinculado a cada objetivo, houve a possibilidade de o gestor do POLO_EAD analisar a categorização dos objetivos e refletir sobre cada elemento anteriormente selecionado e hierarquizado para compor o modelo. Esta categorização é ilustrada na Figura 3 (c). Os itens, justificativa e padrão, foram obtidos junto às entrevistas realizadas com o coordenador do POLO_EAD. Identificada as justificativas e os padrões, passou-se à construção dos indicadores, Figura 3 (d).

Ao estruturar os indicadores o coordenador do polo optou por categorizá-los nos seguintes níveis: deficiente, fraco, regular, bom e muito bom. A opção pela denominação deve-se ao fato de esta ser a nomenclatura utilizada pelo MEC ao se proceder a avaliação dos cursos, ou seja, o coordenador optou por utilizar uma nomenclatura similar. Além da nomenclatura, o coordenador também optou por manter a mesma pontuação atribuída pelo MEC, ou seja, o nível muito bom (MB) refere-se a elementos que foram apresentados ou descritos com uma indicação de desempenho 
equivalente a "5 pontos". Para as demais categorizações bom (B), regular (R), fraco (F) e deficitário (D) foram utilizados critérios idênticos, sendo a eles atribuídos "4 pontos", "3 pontos", "2 pontos" e "1 ponto" respectivamente.

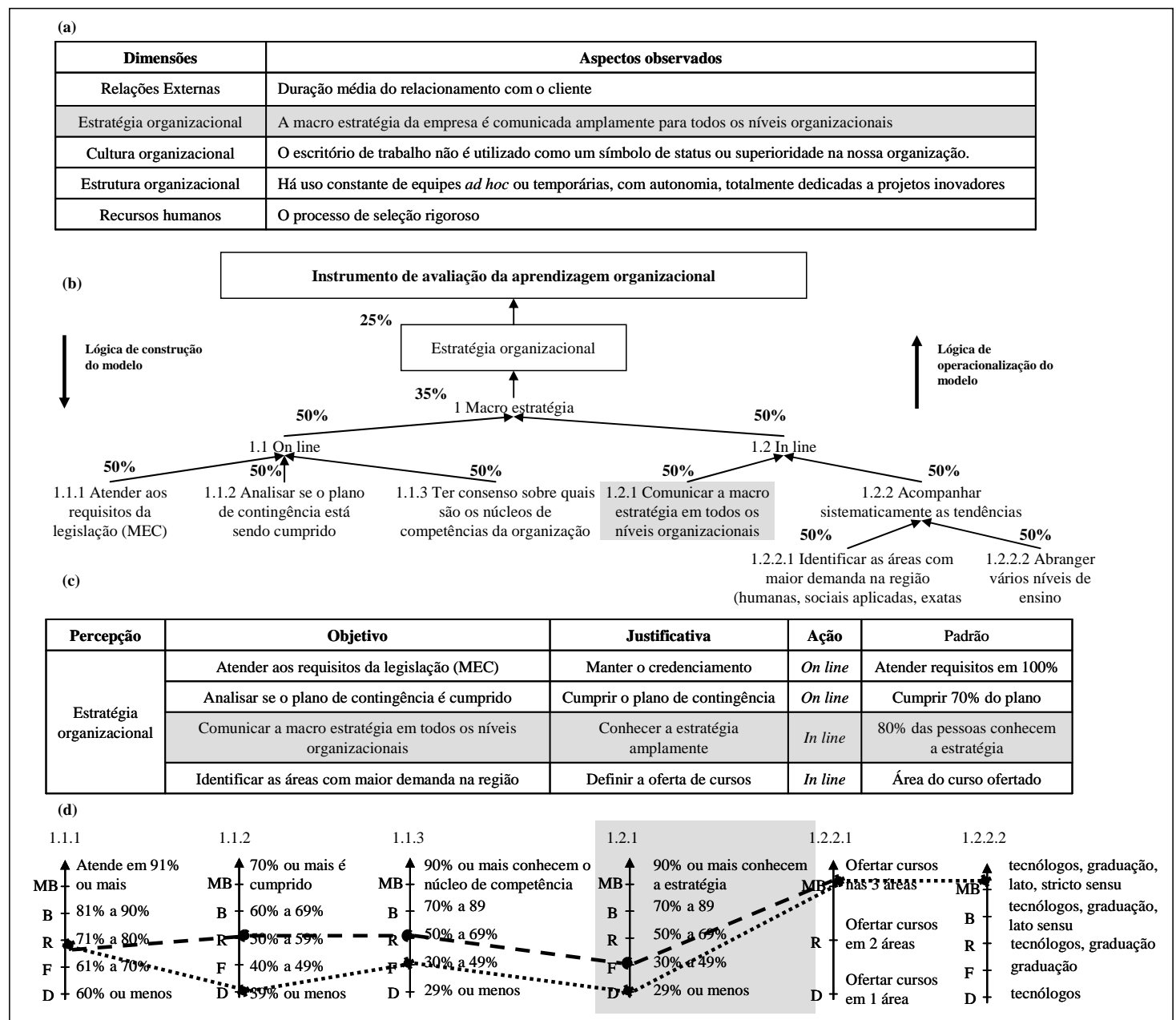

Figura 3: Estruturação e operacionalização do modelo proposto por Igarashi (2009) para o POLO_EAD

Estruturados os indicadores passou-se à definição dos pesos, para se proceder ao agrupamento dos indicadores em termos dos objetivos, ações e percepções. Este processo está previsto no modelo como a última atividade da fase 1.

Os pesos atribuídos a cada um dos elementos que compõem o modelo foram obtidos a partir de entrevistas realizadas com o gestor do POLO_EAD. No processo de identificação dos pesos, foi explicado ao gestor a função dos pesos no modelo, os quais possibilitam o agrupamento dos indicadores, a fim de obter uma avaliação nos diversos níveis ou mesmo global. Outro ponto explicado ao gestor se refere ao processo de atribuição dos pesos. Neste processo foi necessário que o gestor realizasse a análise de cada agrupamento, que compõem o modelo, e identificasse dentro de cada um, quais elementos são mais significativos segundo sua ótica, uma vez que aos elementos mais significativos seriam atribuídos pesos mais elevados. Atribuídos os pesos aos elementos objetos de avaliação encerra-se a fase 1 e se inicia a fase 2 do modelo.

\subsection{Fase 2 - Operacionalização do modelo}

Na fase 2 participaram todos os membros efetivos do polo, sendo realizadas novas entrevistas de modo conjunto e interativo, a fim de identificar o desempenho do 
POLO_EAD em cada um dos indicadores identificados e construídos na fase 1.

É na fase de operacionalização que se torna possível identificar o desempenho do POLO_EAD em nível local (Figura 3 (d) linha pontilhada), ou seja, o desempenho atribuído a cada indicador. Destaca-se que o perfil local identificado a partir das respostas obtidas nas entrevistas realizadas é representado pela linha pontilhada, a qual é traçada ao longo das setas (indicadores). Com a análise, em nível local é possível observar em quais indicadores a organização apresentou um desempenho de maior ou menor impacto. Obtido o perfil de desempenho local, passa-se ao agrupamento da pontuação, até atingir o nível global. Este processo é realizado por meio da relação entre os conceitos e os pesos. O procedimento é ilustrado no Quadro 2, onde foi desdobrado apenas em termos da percepção da Estratégia Organizacional.

Quadro 2: Agrupamento da avaliação local

\begin{tabular}{|c|c|c|c|}
\hline Elementos objetos de avaliação & Pesos & Classif. & Pont. \\
\hline Estratégia Organizacional & $25 \%$ & & 2,41 \\
\hline Macro estratégia & $35 \%$ & & 2,58 \\
\hline On Line & $50 \%$ & & 2,50 \\
\hline Atender aos requisitos da legislação (MEC) & $30 \%$ & $\mathrm{R}$ & 3,00 \\
\hline Analisar se o plano de contingência está sendo cumprido & $40 \%$ & & 1,00 \\
\hline $\begin{array}{l}\text { Ter consenso sobre quais são os núcleos de competências da } \\
\text { organização }\end{array}$ & $30 \%$ & B & 4,00 \\
\hline In line & $50 \%$ & & 2,65 \\
\hline $\begin{array}{l}\begin{array}{l}\text { Comunicar a } \\
\text { organizacionais }\end{array}\end{array}$ & $35 \%$ & $\mathrm{~F}$ & 2,00 \\
\hline Acompanhar sistematicamente as tendências & $65 \%$ & & 3,00 \\
\hline Identificar as áreas com maior demanda na região & $50 \%$ & $\mathrm{D}$ & 1,00 \\
\hline Abranger vários níveis de ensino & $50 \%$ & MB & 5,00 \\
\hline Micro estratégia & $35 \%$ & & 2,68 \\
\hline Resultado & $30 \%$ & & 1,90 \\
\hline
\end{tabular}

A partir do processo de agrupamento realizado, Quadro 2, é possível ao gestor do POLO_EAD realizar a análise do desempenho da organização em diversos níveis de agrupamento. Por exemplo, o gestor pode realizar a análise ao nível das ações nos agrupamentos in-line, on-line ou off-line, ou ainda ao nível das percepções relacionadas à aprendizagem organizacional (estratégia organizacional, infraestrutura organizacional, cultura organizacional, recursos humanos internos e relações externas).

\subsection{Fase 3 - Gerenciar o desempenho}

A fase 3 prevista no modelo contempla a revisão periódica dos indicadores e das metas que compõem o modelo estruturado para o POLO_EAD. Deste modo, o modelo proposto apresenta um ciclo permanente de melhorias ao processo.

Esta etapa até o presente momento foi desenvolvida apenas no que se refere ao curto prazo. Ou seja, foi realizada a primeira avaliação do POLO_EAD e sugeridas ações de melhorias aos desempenhos considerados como críticos (abaixo do nível regular). Contudo, ainda não foi realizada a segunda operacionalização do modelo para identificar se ações efetivamente refletiram os resultados esperados, tão pouco foi realizado o processo de feedback nas diversas fases e etapas. A partir do exposto a fase 3 foi operacionalizada apenas em relação à etapa de análise do desempenho, mais especificamente relativo aos processos: identificar ações de melhoria em curto prazo que possam ser implementadas em nível local, a fim de melhorar o desempenho em nível global; de modo a simular a realimentação das informações e verificar, se ações de 
melhoria permitem a organização melhorar seu desempenho.

O processo de gerenciamento é ilustrado no Quadro 3. Esta etapa resgata o desempenho identificado em nível local, o que torna possível conhecer em quais indicadores avaliados o POLO_EAD apresentou desempenhos considerados críticos pelo gestor.

Quadro 3: Ações de melhoria sugeridas para a alavancagem do desempenho

\begin{tabular}{|l|c|l|c|}
\hline \multicolumn{1}{|c|}{$\begin{array}{c}\text { Indicadores com desempenho em } \\
\text { nível “inferior ao regular” }\end{array}$} & $\begin{array}{c}\text { Situação } \\
\text { atual }\end{array}$ & Ação sugerida & $\begin{array}{c}\text { Nova } \\
\text { situação }\end{array}$ \\
\hline $\begin{array}{l}\text { Atender aos requisitos da legislação } \\
\text { (MEC) }\end{array}$ & R & Não foi objeto de análise & R \\
\hline $\begin{array}{l}\text { Analisar se o plano de contingência é } \\
\text { cumprido }\end{array}$ & D & $\begin{array}{l}\text { Cumprir plano de contingência em } \\
\text { até 40\% no primeiro ano }\end{array}$ & $\mathrm{R}$ \\
\hline $\begin{array}{l}\text { Ter consenso sobre quais são os núcleos } \\
\text { de competências da organização }\end{array}$ & $\mathrm{F}$ & $\begin{array}{l}\text { Realizar reuniões com os membros } \\
\text { da organização para divulgar o } \\
\text { núcleo de competências da mesma }\end{array}$ & $\mathrm{F}$ \\
\hline $\begin{array}{l}\text { Comunicar a macro estratégia em todos } \\
\text { os níveis organizacionais }\end{array}$ & $\mathrm{D}$ & $\begin{array}{l}\text { Realizar reuniões com os } \\
\text { colaboradores para divulgar a } \\
\text { estratégia }\end{array}$ & - \\
\hline $\begin{array}{l}\text { Identificar as áreas com maior demanda } \\
\text { na região }\end{array}$ & MB & Não foi objeto de análise & - \\
\hline Abranger vários níveis de ensino & MB & Não foi objeto de análise & \\
\hline
\end{tabular}

As ações de melhoria apresentadas no Quadro 3, foram identificadas em entrevista realizada com o gestor do POLO_EAD, no momento em que foi entregue o resultado da tabulação (perfil local de desempenho, e agrupamento dos indicadores) das entrevistas anteriores. As ações de melhoria listadas representam algumas das possibilidades de ações no processo de gerenciamento, que pode ser realizado a partir da operacionalização do modelo que aqui se propõe. Neste sentido, o subsídio fundamental para o gerenciamento do POLO_EAD origina-se, exatamente, nos indicadores, vinculados a cada uma das perspectivas. Ao se operacionalizar as melhorias propostas o novo perfil de desempenho pode ser visualizado na Figura 3 (d) pela linha tracejada.

Diante do exposto, salienta-se o efeito advindo da estruturação dos indicadores, para cada percepção relacionada à aprendizagem organizacional. Neste sentido, fica evidenciada a natureza das ações pontuais a serem tomadas, com vistas ao aperfeiçoamento do desempenho do POLO_EAD, uma vez que a visualização do nível de impacto em cada indicador torna-se evidente.

Como consequência torna-se possível, propor ações de aperfeiçoamento. Assim, considera-se que o modelo estruturado nestas bases pode oferecer ao gestor do POLO_EAD, subsídios para um processo personalizado de gerenciamento, calcado nas preocupações e preferências dos envolvidos da organização.

\section{Considerações finais}

A partir da operacionalização do modelo foi possível obter uma melhor compreensão dos procedimentos adotados pela organização no processo de aprendizagem organizacional. Dentre os elementos que apoiaram a melhor compreensão do processo, destaca-se a identificação de cada um dos indicadores que geram a avaliação local e global da organização.

Ao se identificar o desempenho da organização em cada um dos indicadores, tornou-se possível conhecer em quais apresentou desempenhos "abaixo do nível regular”, os quais foram considerados com desempenho crítico e careciam de ações de 
melhoria, ou seja, referem-se a elementos que o gestor deveria direcionar esforços a fim de obter a alavancagem do desempenho. Neste sentido, o modelo proposto auxilia no processo de gestão, ou seja, no gerenciamento da organização.

A partir da identificação do desempenho em termos dos indicadores, o processo de agrupamento dos elementos em objetivos estratégicos in-line, off-line ou on-line, ou seja, operacional, tático e estratégico, proporcionou para a organização objeto de estudo, a identificação de questões que estão vinculadas ao seu planejamento. De modo que o gestor verifique ações que sejam necessárias no processo evolutivo da organização.

Salienta-se que a construção dos indicadores, para cada um dos elementos que compõem o processo de avaliação, possibilitou ao gestor identificar ações pontuais a serem tomadas. Da mesma forma, fica evidenciado o desempenho atual da organização, bem como potencializa a identificação de ações que conduzam ao aperfeiçoamento. Deste modo, o modelo proposto ofereceu à organização, subsídios para o gerenciamento associado aos elementos atuais, ou ainda, a outros elementos que venham a ser incorporados, quer seja, devido às possíveis alterações nas percepções do envolvidos, por necessidade do gestor, ou devido à evolução da organização.

Acredita-se que a partir dos elementos observados, o modelo proposto pode auxiliar no autoconhecimento e no apoio ao gerenciamento das organizações, de modo a contemplar as percepções e preocupações observadas pelos autores pesquisados, bem como considerar as especificidades organizacionais, ao se estruturar o modelo no enfoque e contexto da organização, objeto de estudo. Como continuidade desta pesquisa podem ser realizadas novas operacionalizações do modelo em outras organizações para testar sua robustez, ou ainda a realização de várias avaliações em uma mesma organização a fim de se testar a gestão da melhoria contínua e o feedback.

\section{Referências bibliográficas}

BOLLINGER A., SMITH R. Managing organizational knowledge as a strategic asset. Journal of Knowledge Management, v. 5, n. 1, p. 8 - 18, 2001.

CHAE, B.; LANZARA, G. F. Self-destructive dynamics in large-scale technochange and some ways of counteracting it. Information Technology \& People, v. 19, n. 1, p. $74-97,2006$.

DOVE, R. Knowledge management, response ability, and the agile enterprise. Journal of Knowledge Management, v. 3, n. 1, p. 18 - 35, 1999.

FALCONER, L. Organizational learning, tacit information and e-learning: A review. The Learning Organization, v. 13, n. 2, p. 140 - 151, 2006.

GASSON, S.; SHELFER, K. M. IT-based knowledge management to support organizational learning Visa application screening at the INS. Information Technology \& People, v. 20, n. 4, p. 376 - 399, 2007.

GIL, A. C. Métodos e técnicas de pesquisa social. São Paulo: Atlas, 2002.

HALAWI, L. A.; MCCARTHY, R. V.; ARONSON, J. E. Knowledge management and the competitive strategy of the firm. The Learning Organization: An International Journal, v. 13, n. 4, p. 384 - 397, 2006.

HUOSONG, X., KUANQI, D. and SHUQIN, C., Enterprise knowledge tree model and 
factors of KMS based on E-C. Journal of Knowledge Management, v. 7, p. 96 - 106, 2003.

IGARASHI, D. C. C. ; ENSSLIN, S. R.; PALADINI, E. P.; ENSSLIN, L. Construção de Modelo híbrido para auto-avaliação de um programa de pós-graduação - a fase de estruturação. In: XIII Simpósio de Eng. de Produção - SIMPEP, 2006, Bauru, 2006.

IGARASHI, W. Aprendizagem organizacional: proposta de um modelo de avaliação. Tese (Doutorado) - Programa de Pós-Graduação em Engenharia e Gestão do Conhecimento, Universidade Federal de Santa Catarina, Florianópolis, 2009.

IGARASHI, W., IGARASHI, D.C.C., NAKAYAMA, M.K. Análise do alinhamento entre os elementos de aprendizagem organizacional/ gestão do conhecimento/ tecnologia da informação no contexto nacional e internacional. CINTED-UFRGS, Novas Tecnologias na Educação, v. 7, n. 1, jul., 2009.

LOEBBECKE, C., WAREHAM, J. The impact of E-Business and the Information Society on 'strategy' and 'strategic planning': An assessment of new concepts and challenges. Information Technology and Management, v. 4, 165-182, 2003.

MATHIASSEN L.; POURKOMEYLIAN P. Managing knowledge in a software organization. Journal of Knowledge Management. v. 7, n. 2, p. 63 - 80, 2003.

MCADAM, R.; MASON, B.; MCCRORY, J. Exploring the dichotomies within the tacit knowledge literature: towards a process of tacit knowing in organizations. Journal of Knowledge Management, v. 11, n. 2, p. 43 - 59, 2007.

MEROÑO-CERDAN, A. L.; LOPEZ-NICOLAS, C.; SABATER-SÁNCHEZ, R. Knowledge management strategy diagnosis from KM instruments use. Journal of Knowledge Management, v. 11, n. 2, p. 60 - 72, 2007.

MESO, P; SMITH, R. A Resource-Based View of Organizational Knowledge Management Systems. Journal of Knowledge Managemet, v. 4, n. 3, p. $224-231$, 2000 .

MIKLÓS, G. B. Avaliação do Rendimento Educacional. São Paulo: EPU, 1980.

NEVO, D.; FURNEAUX, B.; WAND, Y. Towards an evaluation framework for knowledge management systems. Information Technology and Management, v. 9 , n. 4, dez., p. 233 - 249, 2007.

OTHMAN, R.; HASHIM, N.A. Typologizing organizational amnesia. The Learning Organization: An International Journal, v. 11, n. 3, p. 273-284, 2004.

RICHARDSON, R. J. Pesquisa social: métodos e técnicas. São Paulo: Atlas, 1999.

SILVA, S. L. Informação e competitividade: a contextualização da gestão do conhecimento nos processos organizacionais. Ci. Inf., v. 31, n. 2, p. 108 - 109, 2002.

SZYMCZAK, C.C.; WALKER, D.H.T. Boeing - a case study example of enterprise project management from a learning organization perspective. The Learning Organization: An International Journal, v. 10, n. 3, p. 125-137, 2003. 